\title{
Linear radial Regge trajectories for mesons with any quark flavor
}

\author{
Sergey Afonin ${ }^{1, \star}$ and Ilya Pusenkov ${ }^{1, \star \star}$ \\ ${ }^{1}$ Saint Petersburg State University, 7/9 Universitetskaya nab., St.Petersburg, 199034, Russia
}

\begin{abstract}
In the Regge phenomenology, the radial spectrum of light mesons is given by a linear relation $M_{n}^{2}=a(n+b)$, where $a$ is a universal slope, the dimensionless intercept $b$ depends on quantum numbers, and $n$ enumerates the excited states in radial recurrences. The usual extensions of this relation to heavy quarkonia in the framework of hadron string models typically lead to strong nonlinearities which seem to be at variance with the available experimental data. Introducing a radially static string picture of mesons, we put forward a linear generalization $\left(M_{n}-m_{1}-m_{2}\right)^{2}=a(n+b)$, where $m_{1,2}$ are quark masses. The vector channel contains enough experimental states to check this new relation and a good agreement is observed. It is shown that this generalization leads to a simple estimate of current quark masses from the radial spectra.
\end{abstract}

The Regge phenomenology grew out of the dual amplitudes [1] and gave rise to various hadron string models. This approach continues to play an important role in the study of hadron spectroscopy. The dual amplitudes and some related string approaches predict the following behavior of meson masses,

$$
M_{n}^{2}=a(J+n+c), \quad J, n=0,1,2, \ldots,
$$

where $J$ is the spin, $n$ enumerates the radially excited states, $a$ represents a universal slope and $c$ is a constant. The relation (1) reproduces the classical Regge behavior $M^{2} \sim J$ observed in the light baryons and mesons. Also this relation predicts the equidistant daughter Regge trajectories. The number $n$ enumerates the states on these "radial" Regge trajectories. The available experimental data on light non-strange mesons seem to confirm the linear Regge behavior (1) [2-5].

Various attempts to generalise the relation (1) to the sector of heavy quarks resulted in the emergence of strong non-linearities with respect to $J$ and $n$. Let us look, however, at the experimental data. A relatively rich set of data on radial recurrences in this sector exists only for the unflavored vector heavy quarkonia. Using the relevant data from the Particle Data [6] which are displayed in Table 1 (see Ref. [7] for detailed discussions on the data choice) we plot the masses squared of known $\omega, \varphi$, $\psi$, and $\Upsilon$-mesons as a function of consecutive number $n$ in Figs. $1-4$. Aside from the ground state, the masses approximately lie on the linear radial trajectories

$$
M_{n}^{2}=a(n+b),
$$

where we re-denoted the vector intercept $b=1+c$. The slope and intercept in (2) depend strongly on the quark flavor, see Table 2.

\footnotetext{
${ }_{\star}^{\star}$ e-mail: s.afonin@spbu.ru

$\star \star$ e-mail: i.pusenkov@spbu.ru
} 
Table 1. The masses of known $\omega, \phi, \psi$ and $\Upsilon$ mesons (in $\mathrm{MeV}$ ) which are used in our analysis. The experimental error is not displayed if it is less than $1 \mathrm{MeV}$.

\begin{tabular}{|c|ccccc|}
\hline$n$ & 0 & 1 & 2 & 3 & 4 \\
\hline$M_{\omega}$ & 783 & $1425 \pm 25$ & $1670 \pm 30$ & $1960 \pm 25$ & $2205 \pm 30$ \\
$M_{\phi}$ & 1020 & $1680 \pm 20$ & - & $2175 \pm 15$ & - \\
$M_{\psi}$ & 3097 & 3686 & $4039 \pm 1$ & $4421 \pm 4$ & - \\
$M_{\Upsilon}$ & 9460 & 10023 & 10355 & $10579 \pm 1$ & $10865 \pm 8$ \\
\hline
\end{tabular}

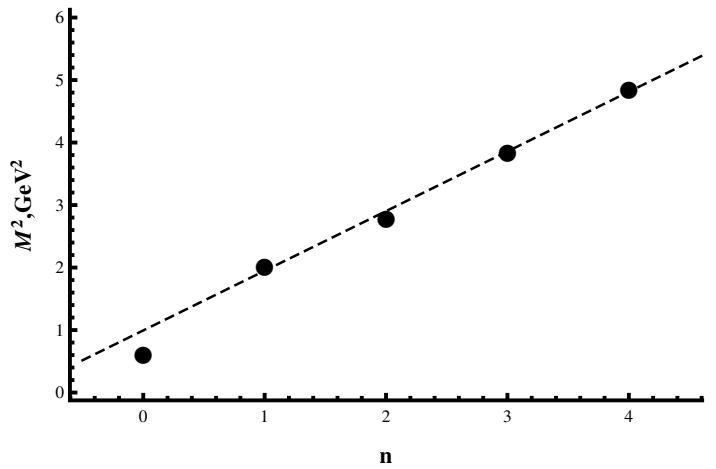

Figure 1. The spectrum of $\omega$-mesons. The experimental points (for this and subsequent figures) are taken from Table 1.

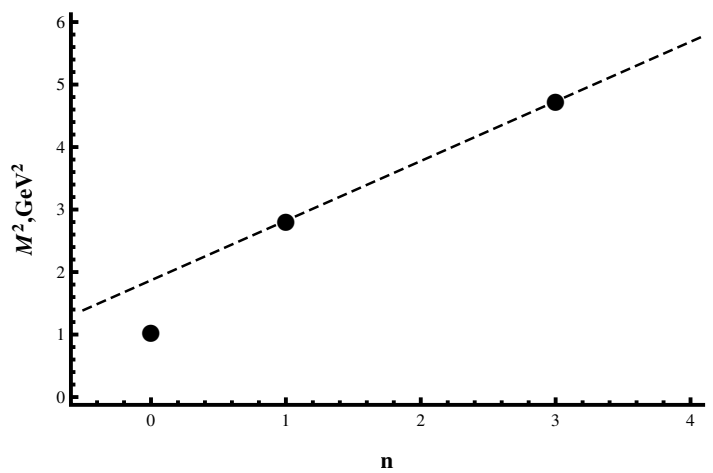

Figure 2. The spectrum of $\phi$-mesons.

The universal linearity seen in Figs. 1 - 4 suggests that some universal gluodynamics lies behind the observed behavior. We propose a simple string scheme explaining this universality. The central idea is that at the conditions when a quark-antiquark pair form a resonance in the QCD vacuum, the pair can be viewed as a radially static system. The binding is provided by the exchange of some massless particle (the pion or gluon in concrete realizations of the scheme). And one must quantize the motion of this particle (not the radial motion of quarks as in the standard hadron string approaches!). The total energy (mass) of the system is

$$
M=m_{1}+m_{2}+p+\sigma r .
$$




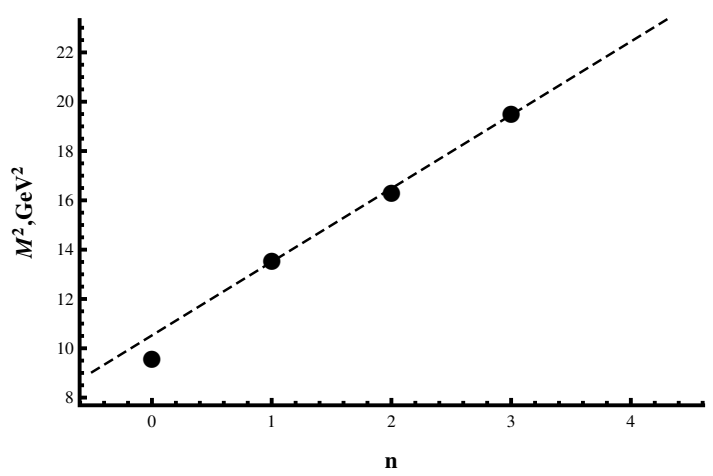

Figure 3. The spectrum of $\psi$-mesons.

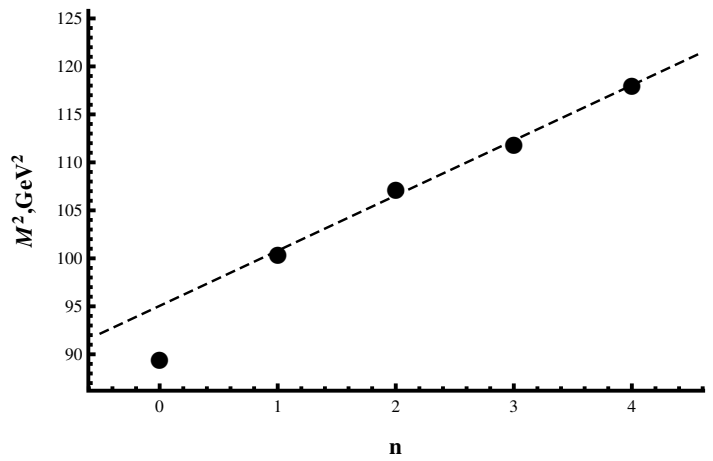

Figure 4. The spectrum of $\Upsilon$-mesons.

Table 2. The radial Regge trajectories (2) (in $\mathrm{GeV}^{2}$ ) for the data from Table 1 (see text).

\begin{tabular}{|c|cc|}
\hline$M_{n}^{2}$ & Fit (a) & Fit (b) \\
\hline$M_{\omega}^{2}$ & $1.03(n+0.74)$ & $0.95(n+1.04)$ \\
$M_{\phi}^{2}$ & $1.19(n+1.07)$ & $0.95(n+1.96)$ \\
$M_{\psi}^{2}$ & $3.26(n+3.03)$ & $2.98(n+3.53)$ \\
$M_{\Upsilon}^{2}$ & $6.86(n+11.37)$ & $5.75(n+16.54)$ \\
\hline
\end{tabular}

Here $m_{1}$ and $m_{2}$ are the masses of quark and antiquark separated by the distance $r, p$ is the momentum of exchanged particle, and $\sigma$ represents the standard string tension. Let us apply the semiclassical quantization to the momentum $p$

$$
\int_{0}^{l} p d r=\pi(n+b), \quad n=0,1,2, \ldots
$$

Here $l$ is the maximal quark separation and the constant $b$ depends on the boundary conditions. Substituting $p$ from (3) to (4) and making use of the definition $\sigma=\frac{M}{l}$ we obtain the linear radial trajectory

$$
\left(M_{n}-m_{1}-m_{2}\right)^{2}=2 \pi \sigma(n+b) .
$$


Table 3. The quark masses (in $\mathrm{GeV}$ ), the slope $a\left(\right.$ in $\mathrm{GeV}^{2}$ ) and the dimensionless intercept parameter $b$ in the relation (6).

\begin{tabular}{|c|cc|}
\hline & Fit I & Fit II \\
\hline$m_{u, d}$ & 0 & 0.36 \\
$m_{s}$ & 0.13 & 0.49 \\
$m_{c}$ & 1.17 & 1.55 \\
$m_{b}$ & 4.33 & 4.69 \\
\hline$a$ & 1.10 & 0.49 \\
\hline$b$ & 0.57 & 0.00 \\
\hline
\end{tabular}

In our unflavored case $m_{1}=m_{2} \equiv m$ and the relation (5) can be simplified to

$$
\left(M_{n}-2 m\right)^{2}=a(n+b),
$$

which is our generalization of the linear spectrum (2) to non-zero quark masses. In this relation, the universal gluodynamics (the slope $a$ ) and dependence on quantum numbers (the dimensionless intercept $b$ ) are clearly separated from the contribution of quark masses. For this reason, the parameters $a$ and $b$ in the relation (6) should be flavor-independent.

Let us test our expectations. We will consider two cases - with the light quark mass set to zero (Fit I) and with all quark masses unfixed (Fit II). The results of interpolation of the data in Table 1 by the ansatz (6) are given in Table 3. The ensuing two variants for the spectrum are depicted in Fig. 5 and Fig. 6. Taking into account a simplicity of the relation (6), the agreement is remarkable.

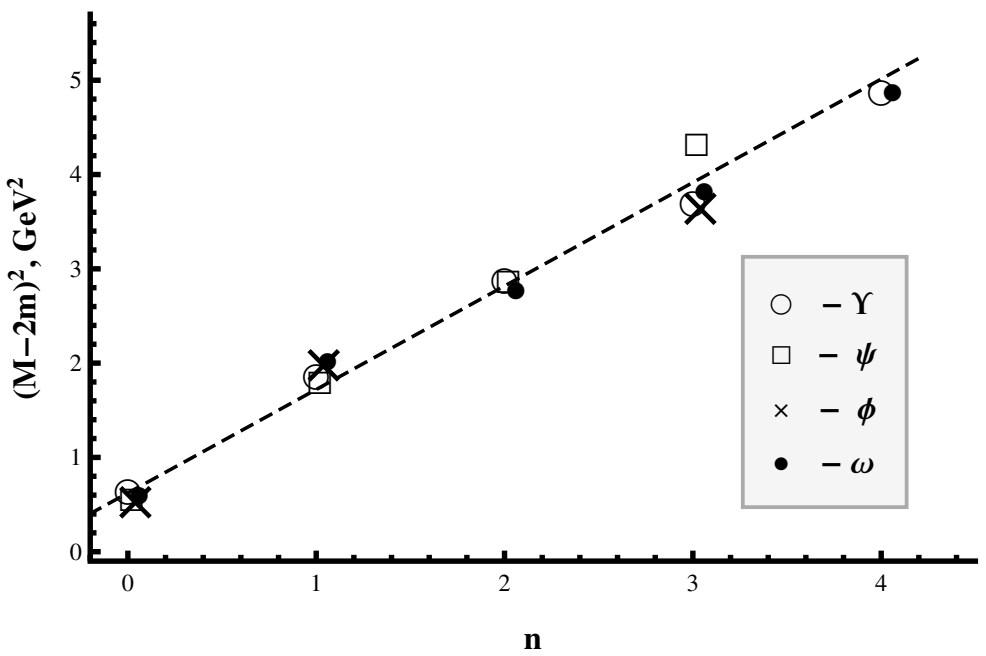

Figure 5. The spectrum (6) for $m_{u, d}$ fixed (Fit I).

The results in Table 3 demonstrate that the radial meson trajectories are able to "measure" the current quark masses with surprisingly good accuracy. If we set $m_{u, d}=0$, the current masses of other quarks turn out to be very close to their phenomenological values [6]. If we keep all quark masses unfixed, they acquire an additional contribution about $360 \mathrm{MeV}$. This contribution may be interpreted as an averaged value of constituent quark mass emerging due to the chiral symmetry breaking in QCD. A more detailed analysis (see Ref. [7]) shows that the Fit I works better and the interpretation with 


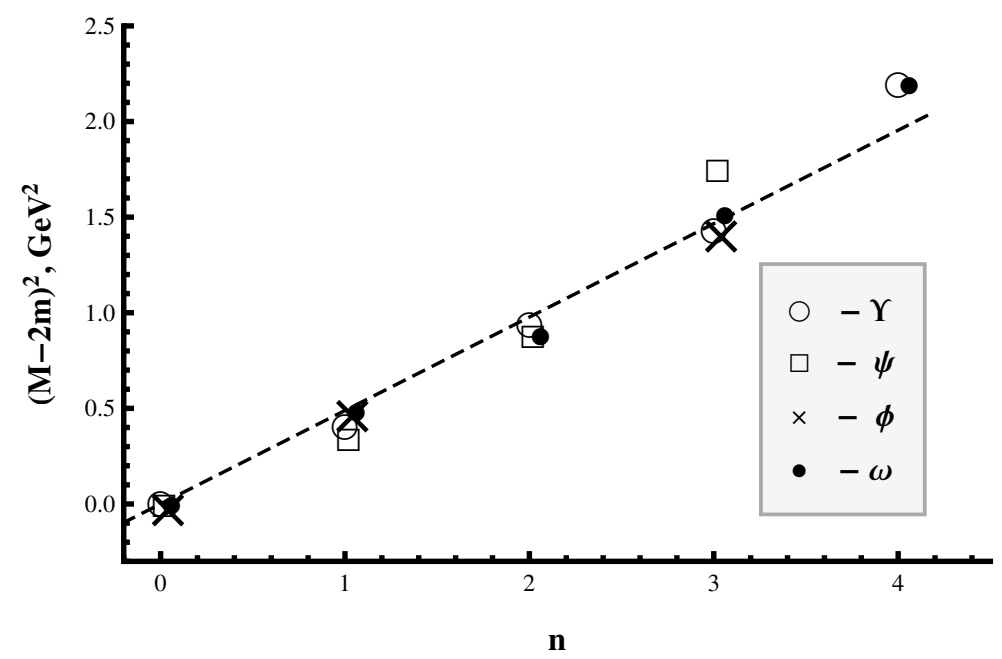

Figure 6. The spectrum (6) for $m_{u, d}$ unfixed (Fit II).

the current quark masses looks preferable. Many related discussions and further fits can be found in Ref. [7]. An independent fit of the relation (6) was also performed by the authors of Ref. [8].

In summary, we proposed a new generalization of linear radial Regge trajectories to the case of massive quarks. Within this generalization, the form of contribution to the meson masses due to confinement is universal for any quarkonia. Our generalization is well consistent with the experimental data on the unflavored vector mesons. Although our considerations were simple and did not take into account various effects which may cause some mass shifts, the quality of final fits is comparable with typical results of semirelativistic potential models, hadron strings and other technically nontrivial approaches.

A natural question emerging after our analysis is whether the relation (6) can be extended to other types of mesons? Unfortunately, the available experimental data are too scarce for making any definite conclusion. A possible extensions of (6) is

$$
\left(M_{n}-m_{1}-m_{2}\right)^{2}=a(n+x+b),
$$

where we may have $x=\beta L$ or $x=\beta J$ (here $L$ and $J$ mean the orbital momentum of valent quarks and total spin). The constant $\beta$ should be fixed from the phenomenology. An intriguing possibility $\beta=1$ would lead to a large degeneracy observed in the light non-strange mesons [3-5]. The fact that the slope $a=2 \pi \sigma$ in (5) coincides with the slope of rotating open string may give a theoretical explanation for this degeneracy.

Acknowledgments. The work was supported by the Saint Petersburg State University research grant 11.38.189.2014 and by the RFBR grant 16-02-00348-a.

\section{References}

[1] P.D.B. Collins, Phys. Rept. C 1, 103 (1971).

[2] A. V. Anisovich, V. V. Anisovich and A. V. Sarantsev, Phys. Rev. D 62, 051502(R) (2000); D. V. Bugg, Phys. Rept. 397, 257 (2004). 
[3] E. Klempt and A. Zaitsev, Phys. Rep. 454, 1 (2007).

[4] S. S. Afonin, Phys. Lett. B 639, 258 (2006); Eur. Phys. J. A 29, 327 (2006).

[5] S. S. Afonin, Phys. Rev. C 76, 015202 (2007); Mod. Phys. Lett. A 22, 1359 (2007); M. Shifman and A. Vainshtein, Phys. Rev. D 77, 034002 (2008).

[6] K. A. Olive et al. (Particle Data Group), Chin. Phys. C 38, 090001 (2014).

[7] S. S. Afonin and I. V. Pusenkov, Phys. Rev. D 90, 094020 (2014); Mod. Phys. Lett. A 29, 1450193 (2014).

[8] P. Masjuan, E. R. Arriola and W. Broniowski, EPJ Web Conf. 73, 04021 (2014). 